\title{
MGMT methylation status: The advent of stratified therapy in glioblastoma?
}

\author{
Peter Hau ${ }^{\mathrm{a}, *}$, Roger Stupp ${ }^{\mathrm{b}}$ and Monika E. Hegi ${ }^{\mathrm{c}}$ \\ ${ }^{a}$ Klinikum der Universität Regensburg, Klinik und Poliklinik für Neurologie am Bezirksklinikum, 93053 \\ Regensburg, Germany \\ E-mail: peterhau@medbo.de \\ ${ }^{\mathrm{b}}$ Multidisciplinary Oncology Center, University Hospital Lausanne (CHUV), 1011 Lausanne, Switzerland \\ E-mail: roger.stupp@chuv.ch \\ ${ }^{\mathrm{c}}$ Laboratory of Tumor Biology and Genetics Department of Neurosurgery, University Hospital Lausanne (CHUV), \\ 1011 Lausanne, and National Center of Competence in Research (NCCR) Molecular Oncology, Swiss Institute for \\ Cancer Research (ISREC), Switzerland \\ E-mail: monika.hegi@chuv.ch
}

\begin{abstract}
Glioblastomas are the most malignant gliomas with median survival times of only 15 months despite modern therapies. All standard treatments are palliative. Pathogenetic factors are diverse, hence, stratified treatment plans are warranted considering the molecular heterogeneity among these tumors. However, most patients are treated with "one fits all" standard therapies, many of them with minor response and major toxicities. The integration of clinical and molecular information, now becoming available using new tools such as gene arrays, proteomics, and molecular imaging, will take us to an era where more targeted and effective treatments may be implemented.

A first step towards the design of such therapies is the identification of relevant molecular mechanisms driving the aggressive biological behavior of glioblastoma. The accumulation of diverse aberrations in regulatory processes enables tumor cells to bypass the effects of most classical therapies available. Molecular alterations underlying such mechanisms comprise aberrations on the genetic level, such as point mutations of distinct genes, or amplifications and deletions, while others result from epigenetic modifications such as aberrant methylation of $\mathrm{CpG}$ islands in the regulatory sequence of genes. Epigenetic silencing of the $M G M T$ gene encoding a DNA repair enzyme was recently found to be of predictive value in a randomized clinical trial for newly diagnosed glioblastoma testing the addition of the alkylating agent temozolomide to standard radiotherapy. Determination of the methylation status of the MGMT promoter may become the first molecular diagnostic tool to identify patients most likely to respond that will allow individually tailored therapy in glioblastoma.

To date, the test for the MGMT-methylation status is the only tool available that may direct the choice for alkylating agents in glioblastoma patients, but many others may hopefully become part of an arsenal to stratify patients to respective targeted therapies within the next years.
\end{abstract}

Keywords: Glioblastoma, predictive factors, personalized therapy, targeted therapy, MGMT

\footnotetext{
${ }^{*}$ Corresponding author: Peter Hau, MD, Laboratory of NeuroOncology, Klinik und Poliklinik für Neurologie der Universität Regensburg am Bezirksklinikum, 93053 Regensburg, Germany. Tel.: +49 941941 0; Fax: +49 9419041 3005; E-mail: peter.hau@medbo.de.
}

\section{Introduction}

Despite the use of combined treatment regimens including resection, radiotherapy, and chemotherapy, the prognosis for patients with glioblastoma, the most malignant form of glioma (WHO grade IV), remains unfavorable $[5,25,40]$. At the time of diagnosis, median 
survival is around 15 months [44], and at tumor recurrence, life expectancy is a few months only [2].

Consequently, individually tailored therapies for patients with high-grade gliomas are an important issue in modern patient management. Efforts are numerous to find factors identifying patients likely to benefit from the treatments considered. The first step to this is to identification of biologically and clinically relevant molecular changes. This has become easier within the last years by new technologies allowing high-throughput analyses in the fields of genomics, epigenomics, and proteomics. However, the true relevance of this descriptive information in the clinical setting depends on its prognostic and predictive value, and the possibility of targeting the respective molecular mechanisms specifically, will translate into efficacious treatments.

In recent years, research has focused on so called epigenetic alterations in tumors. These are heritable changes in gene function that occur without a change in the sequence of nuclear DNA. Epigenetic regulation of genes plays a major role in development [21]. There has been rapid progress in understanding epigenetic mechanisms in cancer, which include aberrant DNA methylation at $\mathrm{CpG}$ islands, as well as changes in chromatin structure mediated by mechanisms such as histone acetylation [18]. Hypermethylation of $\mathrm{CpG}$ islands in the promoter of genes leads to loss of expression, while loss of methylation in normally silenced genes may cause inappropriate expression (e.g. IGF2 gene). Both may have tumor promoting effects. In the course of tumor development, epigenetic silencing is known as an early and important mechanism for inactivation of tumor suppressor genes [9]. Pathways deregulated or inactivated by promoter hypermethylation of key genes comprise the cell cycle, apoptosis, DNArepair, growth factor response, and invasion/cell structure [18]. Similar to other tumor associated molecular changes, epigenetic silencing of cancer relevant genes display typical, tumor type dependent frequencies of such alterations [6].

Efforts to develop drugs to reactivate hypermethylated tumor suppressor genes or to deactivate tumor promoting genes as therapy of cancer are ongoing $[4$, $6,18]$.

\section{The role of MGMT in resistance to alkylating chemotherapy}

Epigenetic silencing of the $O$-6-methylguanine-DNA methyltransferase (MGMT) gene by promoter methy- lation has been recognized as an important factor to predict outcome in glioblastoma patients treated with alkylating agents such as temozolomide (TMZ) and carmustine (BCNU) $[8,16]$. The $M G M T$ gene is located on chromosome 10q26 and codes for an excision repair enzyme removing alkyl-groups from the O6-position of guanine, one of the targets of alkylating agents (Fig. 1). In this process the alkyl group is transferred to the active site of the MGMT protein that thereby becomes irreversibly inactivated and subsequently degraded, requiring resynthesis. Although O6-methyl guanine accounts for less than $10 \%$ of the lesions induced by alkylating agents, it plays a major role as a trigger for cytotoxicity and apoptosis [33]. If left unrepaired, e.g. due to epigenetic silencing of the MGMT gene or depletion of the MGMT protein by saturation of the process, O6methyl guanine persists in the DNA. The current hypothesis proposes that O6-methyl guanine forms mispairs with thymine after replication that are recognized by the mismatch repair (MMR) system. However, since MMR is directed to the newly synthesized strand, while the O6-methyl guanine resides in the template strand, the lesion is not repaired, but engages MMR in what is called futile repair cycles that eventually lead to cell death [23]. Thus, suggesting that in addition to an inactivated MGMT gene, MMR-proficiency is an important factor for response. In line with this mechanism, it has been reported that cells deficient for MMR are 100 times more resistant to alkylating drugs even in absence of MGMT [41].

Taken together, high endogenous MGMT activity in tumors reverses in part the impact of alkylating drugs widely used in oncology [13]. MGMT therefore plays a key role in mechanisms conferring resistance to treatments with alkylating agents, in particular to Temozolomide [11,20,39].

\section{Epigenetic silencing of MGMT predicts benefit from Temozolomide treatment}

The MGMT methylation status was first established as an independent prognostic factor in a phase II trial treating newly diagnosed glioblastoma patients with radiotherapy and concomitant and adjuvant Temozolomide $[16,42]$ and previously for patients treated with nitrosourea chemotherapy $[8,20]$. The subsequent randomized phase III trial enrolling 573 patients conducted by the European Organisation for Research and Treatment of Cancer (EORTC) and the National Cancer Institute of Canada (NCIC) Clinical Trials Group [44] 


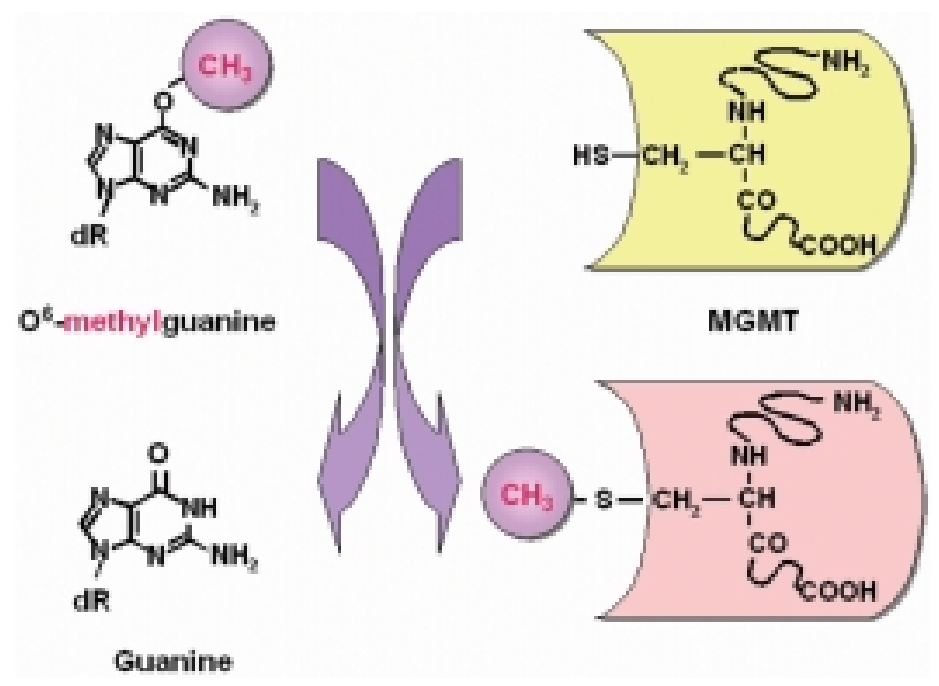

Fig. 1. MGMT mediated repair of $\mathrm{O}^{6}$-methylguanine. The MGMT restores guanine by removing the alkyl-adduct (here represented by a methyl group) from the $\mathrm{O}^{6}$-position of guanine. The transfer of the alkyl-group to the active site of the enzyme inactivates the protein irreversibly and the protein is subjected to degradation [13].

provided the unique opportunity to test the $M G M T$ methylation status as a predictive factor for response to temozolomide treatment. The trial has shown that the addition of concomitant and adjuvant TMZ to radiotherapy (RT) improved the 2-year survival rate from $10 \%$ in the RT group to $26 \%$ for TMZ/RT, setting a new standard of care for glioblastoma patients [42]. In the subgroup of patients with a methylated MGMT promoter the drug proved to be even more active. At 2 years, $46 \%$ of the patients treated with TMZ/RT and whose tumors were $M G M T$ methylated survived, compared to only $14 \%$ for the patients with unmethylated tumors. Promoter methylation status was identified by Methylation Specific PCR (MSP) in the DNA isolated from the tumor tissues of over 200 of the patients. The benefit of the addition of TMZ chemotherapy was basically confined to patients whose tumors had a methylated MGMT promoter [17] (Table 1, Fig. 2).

A confounding factor in the study was that $60 \%$ of the patients randomized to radiation alone received TMZ at progression. When analyzing progression-free survival, the notion that benefit from TMZ is confined to patients with a methylated MGMT is confirmed. A median progression free survival of 10.3 months was observed in patients treated with TMZ and radiation, as opposed to 5.3 months in the radiation only group. The latter is similar to patients with an ummethylated MGMT either treated with radiation alone or the combined treatment [17] (Table 1). These data and mechanistic considerations detailed above strongly suggest that the MGMT promoter status is a predictive factor for benefit from Temozolomide treatment. It is of note that the MGMT methylation status did not correlate with age and that older patients benefited similarly from the treatment depending mainly on their MGMT status.

\section{Other resistance factors}

However, additional tumor resistance mechanisms to TMZ-chemoradiation exist that need to be identified in order to propose additional treatments to those patients who don't respond despite a methylated MGMT status.

Inactivation of the MMR pathway is crucial for mediating the cytotoxic effect of alkylating agents in absence of MGMT. Microsatellite Instability (MSI), a measure for MMR-deficiency, or epigenetic silencing of a $h M L H 1$, which codes for a key component of the MMR-complex, were not detected in glioblastoma of adult patients $[1,6,22,27]$. However, both was identified in few glioblastoma samples of pediatric patients with familial history of cancer [1,22]. Hence, inactivation of MMR function is likely not an important factor for resistance in adult glioblastoma patients. MSI associated with epigenetic inactivation of $h M L H 1$ are frequently found in sporadic colon cancer, cancer of the uterus, and stomach cancer [6]. MSI results from unrepaired slippage of the DNA polymerase during replication of nucleotide repeats that normally is recognized and repaired by MMR.

Recent comprehensive molecular profiling efforts provided evidence for the existence of molecular sub- 
Table 1

Impact of the methylation status of the MGMT promoter on Temozolomide-treatment mediated benefit on survival (with permission from Hegi et al., 2005 [17]). The table shows progression free and overall survival times separated for patients with methylated and unmethylated MGMT promote, respectively

\begin{tabular}{llll}
\hline Randomization & & Total RT & TMZ/RT \\
\hline Number of patients & $N=206$ & $N=100$ & $N=106$ \\
\hline $\begin{array}{l}\text { MGMT methylated } \\
\text { Progression Free Survival }\end{array}$ & $N=92$ & $N=46$ & $N=46$ \\
Median PFS [95\% CI] months & & $5.9[5.3 ; 7.7]$ & $10.3[6.5 ; 14.0]$ \\
6 months PFS rate [95\% CI] (\%) & & $47.8[33.4 ; 62.3]$ & $68.9[55.4 ; 82.4]$ \\
Overall Survival & & $15.3[13.0 ; 20.9]$ & $21.7[17.4 ; 30.4]$ \\
Median survival [95\% CI] months & & $22.7[10.3 ; 35.1]$ & $46.0[31.2 ; 60.8]$ \\
2-yr survival rate [95\% CI] (\%) & $N=114$ & $N=54$ & $N=60$ \\
MGMT unmethylated & & $4.4[3.1 ; 6.0]$ & $5.3[5.0 ; 7.6]$ \\
Progression Free Survival & & $35[22.5 ; 47.9]$ & $40.0[27.6 ; 52.4]$ \\
Median PFS [95\% CI] months & & $11.8[9.7 ; 14.1]$ & $12.7[11.6 ; 14.4]$ \\
6 months PFS rate [95\% CI] (\%) & & $1.9[0.0 ; 5.5]$ & $13.8[4.8 ; 22.7]$ \\
Overall Survival & & & \\
Median survival [95\%CI] months & & & \\
2-yr survival rate [95\% CI] (\%) & & & \\
\hline
\end{tabular}

Legend: CI, confidence interval.

Unmethylated MGMT

\begin{tabular}{lll} 
Randomization: & RT & TMZ/RT \\
\hline Median OS months & 11.8 & 12.7 \\
2-year survival & $<2 \%$ & $13.8 \%$
\end{tabular}

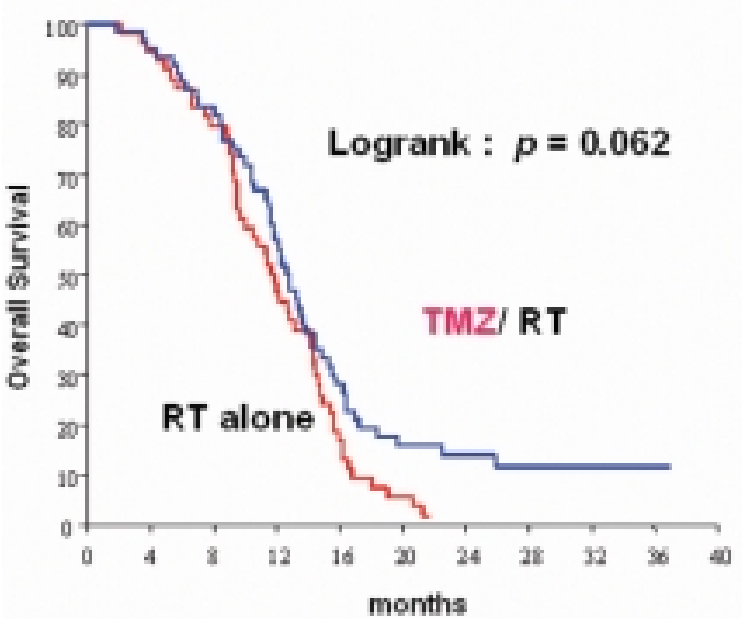

Methylated MGMT

\begin{tabular}{lll} 
Randomization : & $\mathbf{R T}$ & TMZIRT \\
\hline Median OS months & $\mathbf{1 5 . 3}$ & 21.7 \\
2-year survival & $22.7 \%$ & $\mathbf{4 6 . 0} \%$
\end{tabular}

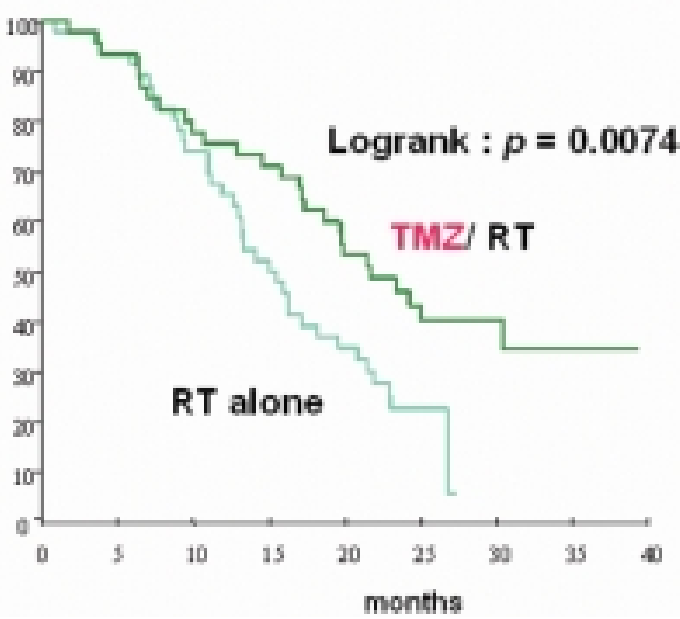

Fig. 2. Overall survival of glioblastoma patients randomized in the EORTC/NCIC-trial according to $M G M T$ methylation status [17,44] Kaplan-Meier survival estimates indicate that the patient group with a methylated $M G M T$ status and randomized to TMZ/RT do best. The patient group with methylated $M G M T$ promoter randomized to RT alone had a favorable overall survival, likely reflecting benefit from TMZ treatment at recurrence (Reproduced with permission. Copyrigh(c) 2005, Massachusetts Medical Society. All rights reserved.).

types of glioblastoma that may differ in their clinical behavior [3,10,14,19,26,31,32,34,36,37]. Several prognostic factors emerged from these and other retrospective studies, however, the insights gained will require validation in independent data sets and prospective clinical trials to determine their predictive value for response to therapeutic interventions. 


\section{Implementation of the MGMT test in routine diagnostics - are we there yet?}

Determination of MGMT methylation status by MSP is an important step towards molecular diagnostics. It provides the opportunity to identify patients likely to benefit from TMZ treatment. Ongoing clinical trials comprising temozolomide as part of the treatment regimen need to stratify for the $M G M T$ status when testing the addition of targeted, small molecule drugs such as inhibitors of tyrosine kinases or integrins implicated in tumor growth and angiogenesis. Preselecting patients by MGMT methylation status will increase patient homogeneity.

The impact of the MGMT methylation status in glioblastoma requires validation. A large randomized international intergroup trial (RTOG 0525/EORTC 26052-22053) has opened in January 2006. It will treat the patients according to the new standard of care and stratify the patients for the MGMT methylation status. This will allow validation of the previous finding, while attempting to overcome MGMT based tumor resistance in the experimental arm by providing a dose dense, intensified temozolomide schedule in the maintenance part, expected to deplete MGMT in the tumor tissue (see outline and treatment schedule in the recent review by Stupp et al. [43]). The MGMT status will be determined with a direct real-time fluorescence-based MSP (Califice, manuscript submitted) that has been adjusted to and validated with the gel based assay reported previously [17]. This new assay will allow high throughput analysis and due to its quantitative nature may allow adjustment of the cut-off for methylated versus non-methylated $M G M T$ at the end of the clinical trial to improve outcome prediction.

At present, for patients with an unmethylated $M G M T$ promoter, the addition of TMZ increases the median survival by less than one month from 11.8 to 12.7 (logrank test $P=0.06$ ) (Table 1, Fig. 2). These patients may profit from other therapies based on different mechanisms of action, and may be spared the toxicity of TMZ. However, no other efficacious treatments are currently available. Nevertheless, patients may be demanding the test in order to decide for or against chemotherapy, or to seek participation in a clinical trial evaluating agents with a different mechanism of action. Until fully validated, $M G M T$ testing may be further indicated in specific clinical circumstances. In patients developing toxicity to the treatment or with a deteriorating performance status, the knowledge of the MGMT promoter methylation status may incite continuation or discontinuation of treatment.

In the future, specific treatments need to be designed and offered to patients with an unmethylated MGMT. Beside other modes of action, strategies comprise the use of specific inhibitors of MGMT, such as the nontoxic substrate O6-benzyl guanine depleting cells of MGMT, expected to render tumors more sensitive to alkylating agent chemotherapy [35]. Other substances modulating MGMT expression may also improve response to alkylating agents $[30,45]$.

\section{Evaluation of the MGMT status}

The presented correlation between MGMT and prediction of benefit from Temozolomide treatment is based on the determination of the methylation status of the MGMT gene promoter in the tumor tissue [17]. Attempts are made to evaluate MGMT at the protein level, or testing MGMT activity in the tumor tissue. For diagnostic purposes the advantage of methylation specific PCR lies in the fact that the methylated MGMT allele is contributed solely by the tumor tissue, and nontumoral tissue contamination does not interfere with the result [38]. Furthermore, MGMT may be induced in tumor cells not only in response to DNA damage introduced by alkylating agents and radiotherapy but also by corticosteroids $[12,15]$. Therefore, since aberrant methylation of $\mathrm{CpGs}$ is an inheritable change of DNA in the clonal expansion of the tumor [46], the methylation status of the MGMT promoter may be a more reliable marker predicting inducibility of MGMT once the cancer treatment is initiated than MGMT levels determined at the time of diagnosis [12,24]. Moreover, MSP yields a clear read-out. However, the test requires good quality paraffin embedded tissue that is not overfixed. In contrast, immunohistochemistry or enzymatic methods may be obscured by the presence of non-tumoral cells and tumor infiltrating lymphocytes in the biopsy that may express MGMT, and retain an unmethylated $M G M T$ promoter status. In our experience, evaluation of immunohistochemistry for MGMT is prone to strong interobserver discrepancies that are even more pronounced when comparing readings from different centers (unpublished results).

\section{Extrapolation to other tumor types}

In clinical trials evaluating TMZ for lower grade glioma and other tumor types such as melanoma and 
NSCLC, the relationship of the MGMT methylation status and response to alkylating chemotherapy has not yet been established, while it had been associated with better prognosis in lymphoma patients treated with alkylating agents [7]. It will be of particular interest to evaluate the relevance of MGMT methylation in oligodendroglioma. In oligodendroglioma with $\mathrm{LOH}$ $1 \mathrm{p} / 19 \mathrm{q}$, a subtype considered particularly sensititve to chemotherapy, MGMT promoter methylation was detected at a frequency of almost $90 \%$ [29].

\section{Conclusions}

Determination of the MGMT methylation status by MSP is an important step towards molecular diagnostics. It provides the opportunity to identify patients likely to benefit from TMZ treatment. These exciting results have moved the field and will incite new research aiming at identifying tumor specific prognostic and predictive molecular characteristics, a prerequisite for individually tailored therapy. For new trials the collection of tumor tissue, best a paraffin block and snap frozen tissue, and other relevant biological material should be mandatory. Subsequent molecular analysis will allow insights into molecular mechanisms underlying success or failure of the treatment strategy. In either case this information will be invaluable for further improvements that are definitely needed.

In the future, tumors may undergo molecular profiling for the identification of the individually altered key pathways that point to the most beneficial therapeutic modality for the respective patient. Response to therapy may be monitored using molecular imaging probes and/or serum biomarkers [28].

\section{References}

[1] M. Alonso, R. Hamelin, M. Kim, K. Porwancher, T. Sung, P. Parhar, D.C. Miller and E.W. Newcomb, Microsatellite instability occurs in distinct subtypes of pediatric but not adult central nervous system tumors, Cancer Res 61 (2001), 21242128.

[2] A.A. Brandes and M.V. Fiorentino, The role of chemotherapy in recurrent malignant gliomas: an overview, Cancer Invest 14 (1996), 551-559.

[3] M. Bredel, C. Bredel, D. Juric, G.E. Duran, R.X. Yu, G.R. Harsh, H. Vogel, L.D. Recht, A.C. Scheck and B.I. Sikic, Tumor necrosis factor-alpha-induced protein 3 as a putative regulator of nuclear factor-kappaB-mediated resistance to O6alkylating agents in human glioblastomas, J Clin Oncol $\mathbf{2 4}$ (2006), 274-287. Epub 2005 Dec 19.
[4] J.F. Costello, M.C. Fruhwald, D.J. Smiraglia, L.J. Rush, G.P. Robertson, X. Gao, F.A. Wright, J.D. Feramisco, P. Peltomaki, J.C. Lang, D.E. Schuller, L. Yu, C.D. Bloomfield, M.A. Caligiuri, A. Yates, R. Nishikawa, H. Su Huang, N.J. Petrelli, X. Zhang, M.S. O'Dorisio, W.A. Held, W.K. Cavenee and C. Plass, Aberrant CpG-island methylation has non-random and tumour-type-specific patterns, Nat Genet 24 (2000), 132-138.

[5] F.G. Davis, S. Freels, J. Grutsch, S. Barlas and S. Brem, Survival rates in patients with primary malignant brain tumors stratified by patient age and tumor histological type: an analysis based on Surveillance, Epidemiology, and End Results (SEER) data, 1973-1991, J Neurosurg 88 (1998), 1-10.

[6] M. Esteller, P.G. Corn, S.B. Baylin and J.G. Herman, A gene hypermethylation profile of human cancer, Cancer Res 61 (2001), 3225-3229.

[7] M. Esteller, G. Gaidano, S.N. Goodman, V. Zagonel, D. Capello, B. Botto, D. Rossi, A. Gloghini, U. Vitolo, A. Carbone, S.B. Baylin and J.G. Herman, Hypermethylation of the DNA repair gene $\mathrm{O}(6)$-methylguanine DNA methyltransferase and survival of patients with diffuse large B-cell lymphoma, $J$ Natl Cancer Inst 94 (2002), 26-32.

[8] M. Esteller, J. Garcia-Foncillas, E. Andion, S.N. Goodman, O.F. Hidalgo, V. Vanaclocha, S.B. Baylin and J.G. Herman, Inactivation of the DNA-repair gene MGMT and the clinical response of gliomas to alkylating agents, $N$ Engl J Med 343 (2000), 1350-1354.

[9] M.F. Fraga, M. Herranz, J. Espada, E. Ballestar, M.F. Paz, S. Ropero, E. Erkek, O. Bozdogan, H. Peinado, A. Niveleau, J.H. Mao, A. Balmain, A. Cano and M. Esteller, A mouse skin multistage carcinogenesis model reflects the aberrant DNA methylation patterns of human tumors, Cancer Res 64 (2004), 5527-5534.

[10] W.A. Freije, F.E. Castro-Vargas, Z. Fang, S. Horvath, T. Cloughesy, L.M. Liau, P.S. Mischel and S.F. Nelson, Gene expression profiling of gliomas strongly predicts survival, Cancer Res 64 (2004), 6503-6510.

[11] H.S. Friedman, R.E. McLendon, T. Kerby, M. Dugan, S.H. Bigner, A.J. Henry, D.M. Ashley, J. Krischer, S. Lovell, K. Rasheed, F. Marchev, A.J. Seman, I. Cokgor, J. Rich, E. Stewart, O.M. Colvin, J.M. Provenzale, D.D. Bigner, M.M. Haglund, A.H. Friedman and P.L. Modrich, DNA mismatch repair and O6-alkylguanine-DNA alkyltransferase analysis and response to Temodal in newly diagnosed malignant glioma, $J$ Clin Oncol 16 (1998), 3851-3857.

[12] G. Fritz, K. Tano, S. Mitra and B. Kaina, Inducibility of the DNA repair gene encoding O6-methylguanine-DNA methyltransferase in mammalian cells by DNA-damaging treatments, Mol Cell Biol 11 (1991), 4660-4668.

[13] S.L. Gerson, MGMT: its role in cancer aetiology and cancer therapeutics, Nat Rev Cancer 4 (2004), 296-307.

[14] S. Godard, G. Getz, M. Delorenzi, P. Farmer, H. Kobayashi, I. Desbaillets, M. Nozaki, A.C. Diserens, M.F. Hamou, P.Y. Dietrich, L. Regli, R.C. Janzer, P. Bucher, R. Stupp, N. de Tribolet, E. Domany and M.E. Hegi, Classification of human astrocytic gliomas on the basis of gene expression: a correlated group of genes with angiogenic activity emerges as a strong predictor of subtypes, Cancer Res 63 (2003), 6613-6625.

[15] T. Grombacher, S. Mitra and B. Kaina, Induction of the alkyltransferase (MGMT) gene by DNA damaging agents and the glucocorticoid dexamethasone and comparison with the response of base excision repair genes, Carcinogenesis $\mathbf{1 7}$ (1996), 2329-2336.

[16] M.E. Hegi, A.-C. Diserens, S. Godard, P.Y. Dietrich, L. Regli, S. Ostermann, P. Otten, G. Van Melle, N. de Tribolet 
and R. Stupp, Clinical trial substantiates the predictive value of MGMT-methylation in glioblastoma patients treated with temozolomide, Clin Cancer Res 10 (2004), 1871-1874.

[17] M.E. Hegi, A.C. Diserens, T. Gorlia, M.F. Hamou, N. de Tribolet, M. Weller, J.M. Kros, J.A. Hainfellner, W.P. Mason, L. Mariani, J.E.C. Bromberg, P. Hau, R.O. Mirimanoff, G. Cairncross, R. Janzer and R. Stupp, MGMT gene silencing and benefit from temozolomide in glioblastoma, New Engl J Med 352 (2005), 997-1003.

[18] J.G. Herman and S.B. Baylin, Gene silencing in cancer in association with promoter hypermethylation, $N$ Engl J Med 349 (2003), 2042-2054.

[19] Y. Iwadate, T. Sakaida, T. Hiwasa, Y. Nagai, H. Ishikura, M. Takiguchi and A. Yamaura, Molecular classification and survival prediction in human gliomas based on proteome analysis, Cancer Res 64 (2004), 2496-2501.

[20] K.A. Jaeckle, H.J. Eyre, J.J. Townsend, S. Schulman, H.M. Knudson, M. Belanich, D.B. Yarosh, S.I. Bearman, D.J. Giroux and S.C. Schold, Correlation of tumor O6 methylguanine-DNA methyltransferase levels with survival of malignant astrocytoma patients treated with bischloroethylnitrosourea: a Southwest Oncology Group study, J Clin Oncol 16 (1998), 3310-3315.

[21] R. Jaenisch and A. Bird, Epigenetic regulation of gene expression: how the genome integrates intrinsic and environmental signals, Nat Genet 33 (2003), 245-254.

[22] M. Kanamori, H. Kon, T. Nobukuni, S. Nomura, K. Sugano, S. Mashiyama, T. Kumabe, T. Yoshimoto, M. Meuth, T. Sekiya and Y. Murakami, Microsatellite instability and the PTEN1 gene mutation in a subset of early onset gliomas carrying germline mutation or promoter methylation of the hMLH1 gene, Oncogene 19 (2000), 1564-1571.

[23] P. Karran, Mechanisms of tolerance to DNA damaging therapeutic drugs, Carcinogenesis 22 (2001), 1931-1937.

[24] G. Kitange, B.L. Carlson, J. LaMont, M. Schroeder, J.N Sarkaria and C.D. James, Use of an orthotopic xenograft model and corresponding xenograft cell cultures to evaluate MGMT promoter hypermethylation and temozolomide response in glioblastoma, Proc Amer Assoc Cancer Res 47 (2006), [Abstract \#5676].

[25] P. Kleihues, D.N. Louis, B.W. Scheithauer, L.B. Rorke, G. Reifenberger, P.C. Burger and W.K. Cavenee, The WHO classification of tumors of the nervous system, J Neuropathol Exp Neurol 61 (2002), 215-225; discussion 226-229.

[26] Y. Liang, M. Diehn, N. Watson, A.W. Bollen, K.D. Aldape, M.K. Nicholas, K.R. Lamborn, M.S. Berger, D. Botstein, P.O. Brown and M.A. Israel, Gene expression profiling reveals molecularly and clinically distinct subtypes of glioblastoma multiforme, Proc Natl Acad Sci USA 102 (2005), 5814-5819. Epub 2005 Apr 12.

[27] R. Martinez, H.K. Schackert, J. Plaschke, G. Baretton, H. Appelt and G. Schackert, Molecular mechanisms associated with chromosomal and microsatellite instability in sporadic glioblastoma multiforme, Oncology (2004), 395-403.

[28] P.S. Mischel, T.F. Cloughesy and S.F. Nelson, DNAmicroarray analysis of brain cancer: molecular classification for therapy, Nat Rev Neurosci 5 (2004), 782-792.

[29] M. Mollemann, M. Wolter, J. Felsberg, V.P. Collins and G. Reifenberger, Frequent promoter hypermethylation and low expression of the MGMT gene in oligodendroglial tumors, Int J Cancer 113 (2005), 379-385.

[30] A. Natsume, D. Ishii, T. Wakabayashi, T. Tsuno, H. Hatano, M. Mizuno and J. Yoshida, IFN-beta down-regulates the expression of DNA repair gene MGMT and sensitizes resistant glioma cells to temozolomide, Cancer Res 65 (2005), 7573 7579 .

[31] J.M. Nigro, A. Misra, L. Zhang, I. Smirnov, H. Colman, C. Griffin, N. Ozburn, M. Chen, E. Pan, D. Koul, W.K. Yung, B.G. Feuerstein and K.D. Aldape, Integrated array-comparative genomic hybridization and expression array profiles identify clinically relevant molecular subtypes of glioblastoma, Cancer Res 65 (2005), 1678-1686.

[32] C.L. Nutt, D.R. Mani, R.A. Betensky, P. Tamayo, J.G. Cairncross, C. Ladd, U. Pohl, C. Hartmann, M.E. McLaughlin, T.T. Batchelor, P.M. Black, A. von Deimling, S.L. Pomeroy, T.R. Golub and D.N. Louis, Gene expression-based classification of malignant gliomas correlates better with survival than histological classification, Cancer Res 63 (2003), 1602-1607.

[33] K. Ochs and B. Kaina, Apoptosis induced by DNA damage O6-methylguanine is Bcl-2 and caspase-9/3 regulated and Fas/caspase-8 independent, Cancer Res 60 (2000), 58155824.

[34] H.S. Phillips, S. Kharbanda, R. Chen, W.F. Forrest, R.H. Soriano, T.D. Wu, A. Misra, J.M. Nigro, H. Colman, L. Soroceanu, P.M. Williams, Z. Modrusan, B.G. Feuerstein and K. Aldape, Molecular subclasses of high-grade glioma predict prognosis, delineate a pattern of disease progression, and resemble stages in neurogenesis, Cancer Cell 9 (2006), 157-173.

[35] J.A. Quinn, A. Desjardins, J. Weingart, H. Brem, M.E. Dolan, S.M. Delaney, J. Vredenburgh, J. Rich, A.H. Friedman, D.A. Reardon, J.H. Sampson, A.E. Pegg, R.C. Moschel, R. Birch, R.E. McLendon, J.M. Provenzale, S. Gururangan, J.E. Dancey, J. Maxwell, S. Tourt-Uhlig, J.E. Herndon, 2nd, D.D. Bigner and H.S. Friedman, Phase I trial of temozolomide plus O6benzylguanine for patients with recurrent or progressive malignant glioma, J Clin Oncol 23 (2005), 7178-7187.

[36] J.N. Rich, C. Hans, B. Jones, E.S. Iversen, R.E. McLendon, B.K. Rasheed, A. Dobra, H.K. Dressman, D.D. Bigner, J.R. Nevins and M. West, Gene expression profiling and genetic markers in glioblastoma survival, Cancer Res 65 (2005), 4051-4058.

[37] S.A. Schwartz, R.J. Weil, R.C. Thompson, Y. Shyr, J.H. Moore, S.A. Toms, M.D. Johnson and R.M. Caprioli, Proteomic-based prognosis of brain tumor patients using direct-tissue matrix-assisted laser desorption ionization mass spectrometry, Cancer Res 65 (2005), 7674-7681.

[38] L. Shen, Y. Kondo, G.L. Rosner, L. Xiao, N.S. Hernandez, J. Vilaythong, P.S. Houlihan, R.S. Krouse, A.R. Prasad, J.G. Einspahr, J. Buckmeier, D.S. Alberts, S.R. Hamilton and J.P. Issa, MGMT promoter methylation and field defect in sporadic colorectal cancer, J Natl Cancer Inst 97 (2005), 1330-1338.

[39] J.R. Silber, A. Blank, M.S. Bobola, S. Ghatan, D.D. Kolstoe and M.S. Berger, O6-methylguanine-DNA methyltransferasedeficient phenotype in human gliomas: frequency and time to tumor progression after alkylating agent-based chemotherapy, Clin Cancer Res 5 (1999), 807-814.

[40] L.A. Stewart, Chemotherapy in adult high-grade glioma: a systematic review and meta-analysis of individual patient data from 12 randomised trials, Lancet 359 (2002), 1011-1018.

[41] L. Stojic, R. Brun and J. Jiricny, Mismatch repair and DNA damage signalling, DNA Repair (Amst) 3 (2004), 1091-1101.

[42] R. Stupp, P.-Y. Dietrich, S. Ostermann Kraljevic, A. Pica, I. Maillard, P. Maeder, R. Meuli, R. Janzer, G. Pizzolato, R. Miralbell, F. Porchet, L. Regli, N. de Tribolet, R.O. Mirimanoff and S. Leyvraz, Promising survival for patients with newly diagnosed glioblastoma multiforme treated with concomitant radiation plus temozolomide followed by adjuvant temozolomide, J Clin Oncol 20 (2002), 1375-1382. 
[43] R. Stupp, M.E. Hegi, M.J. van den Bent, W.P. Mason, M. Weller, R.O. Mirimanoff and J.G. Cairncross, Changing paradigms-an update on the multidisciplinary management of malignant glioma, Oncologist 11 (2006), 165-180.

[44] R. Stupp, W.P. Mason, M.J. van den Bent, M. Weller, B. Fisher, M.J.B. Taphoorn, K. Belanger, A.A. Brandes, J.G. Cairncross, C. Marosi, U. Bogdahn, J. Curschmann, R.C. Janzer, S. Ludwin, T. Gorlia, A. Allgeier, D. Lacombe, E.A. Eisenhauer and R.O. Mirimanoff, Radiotherapy plus concomitant and adjuvant temozolomide for glioblastoma, $N$ Engl J Med 352 (2005), 987-996.

[45] S. Tanaka, I. Kobayashi, S. Utsuki, H. Oka, Y. Yasui and K. Fujii, Down-regulation of O6-methylguanine-DNA methyltransferase gene expression in gliomas by platinum compounds, Oncol Rep 14 (2005), 1275-1280.

[46] A.H. Ting, K.W. Jair, K.E. Schuebel and S.B. Baylin,
Differential requirement for DNA methyltransferase 1 in maintaining human cancer cell gene promoter hypermethylation, Cancer Res 66 (2006), 729-735. 


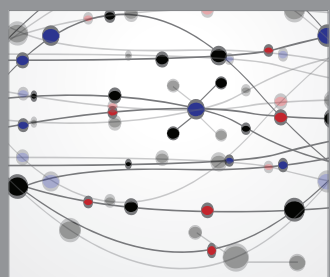

The Scientific World Journal
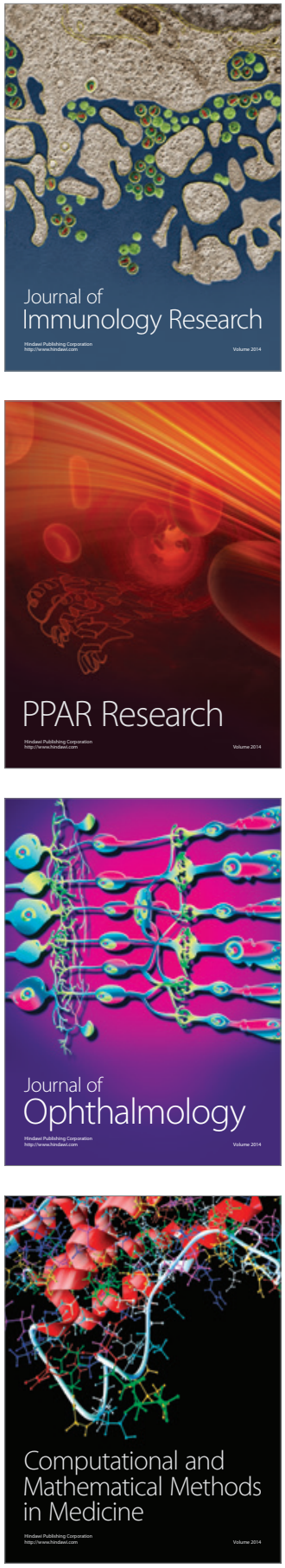

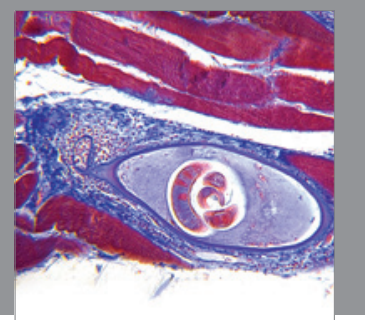

Gastroenterology

Research and Practice
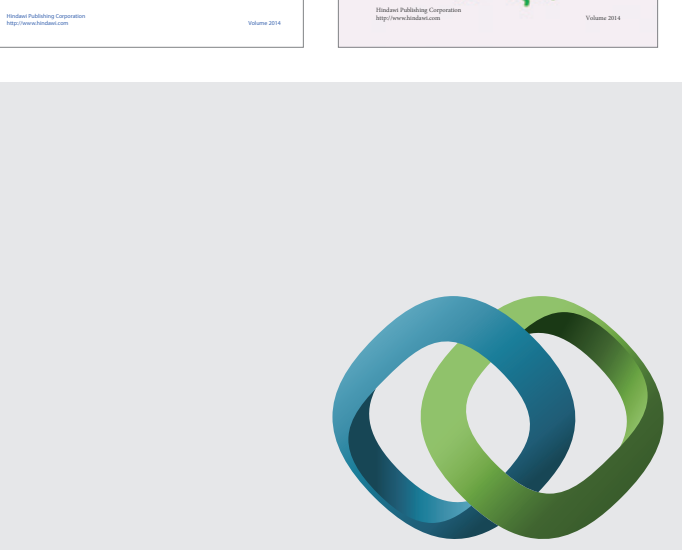

\section{Hindawi}

Submit your manuscripts at

http://www.hindawi.com
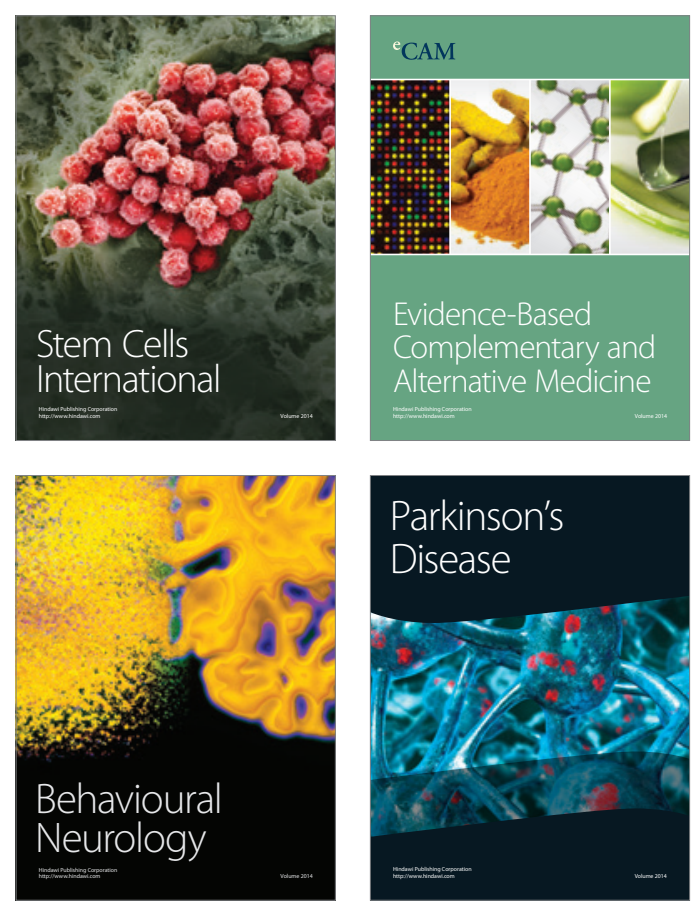

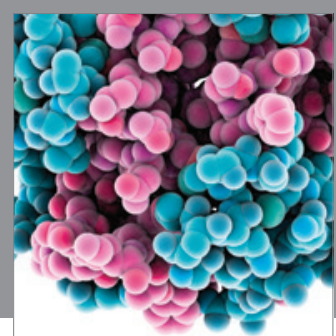

Journal of
Diabetes Research

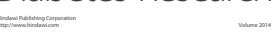

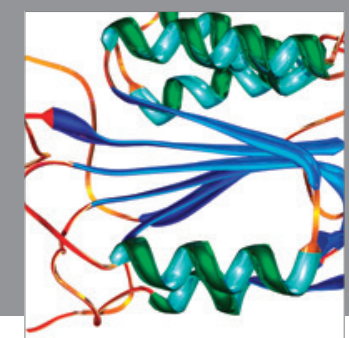

Disease Markers
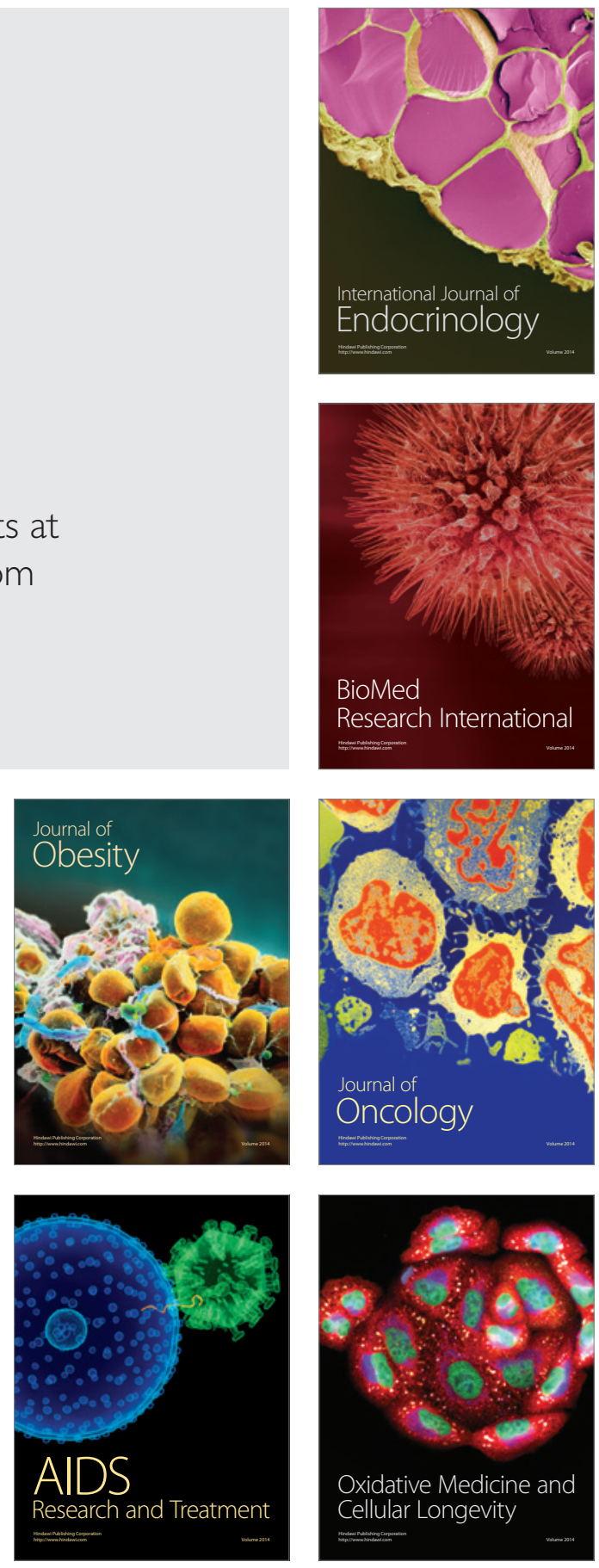